\title{
Ordering of Small Molecules on Hydrophobic Self-Assembled
}

\author{
n-Alkanethiols: Delicate Balance of Interfacial and \\ Intermolecular Interactions
}

\author{
Mithun Ghosh, Ding-Shyue Yang* \\ Department of Chemistry, University of Houston, Houston, Texas 77204, United States
}

*To whom correspondence should be addressed. Email: yang@uh.edu 


\section{ABSTRACT}

Growth of molecular thin films with desired orders and orientations has become technologically relevant as the electronic industries seek new opportunities and applications. However, the delicate balance of interfacial and intermolecular forces and their complex influence on thin-film growths still require more understanding. Here, the effects of a hydrophobic self-assembled monolayer (SAM) surface on the crystallization of four common solvents - acetonitrile, ethanol, methanol, and water-are investigated. Despite the absence of significant substrate-molecule forces, unexpected oriented growth is observed for these molecules except water. Acetonitrile and ethanol form a sustaining vertical assembly order with long-range crystalline structures. Coincident epitaxy with small lattice mismatches is found to be essential to these orderings, which are energetically favored but without a dominant azimuthal orientation. In contrast, a preferred in-plane registry of methanol overlayers is observed for an ultrathin nominal thickness and becomes lost in slightly thicker films. Such thickness-dependent ordering of methanol assemblies can be explained with semi-commensurate epitaxy with a tensile strain of $\sim 6.6 \%$ along hydrogen-bonded chains, whose quick relief results in the loss of order and even the phase. These rich observations suggest that SAM surfaces offer good opportunities for selective crystallization of molecular films worthy of further investigations.

Keywords: amorphous-to-crystalline phase transition, two-dimensional films, van der Waals, strain relief, nucleation

TOC Figure:

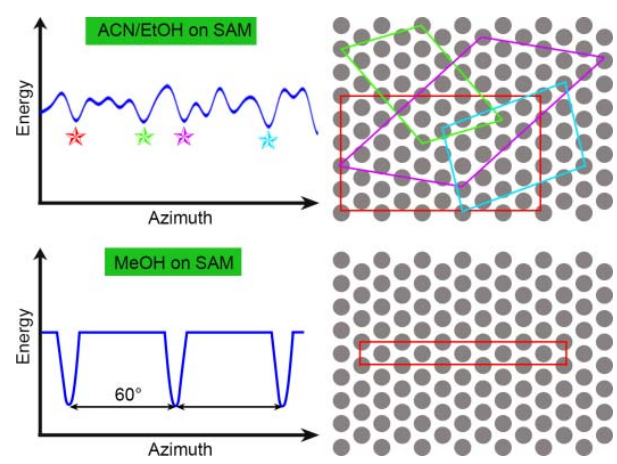




\section{INTRODUCTION}

Crystallization of molecules or materials on solid surfaces has been of significant importance from both fundamental and technological viewpoints. For the latter, emergent applications may be found in various (opto)electronic devices that incorporate, e.g., self-assembled molecular films, ${ }^{1-3}$ liquid crystals, ${ }^{4-7}$ organic photovoltaics, ${ }^{7-9}$ electrode materials, ${ }^{10}$ light-emitting diodes, ${ }^{11-14}$ or field-effect transistors. ${ }^{10,15}$ Certain crystalline phases or morphological forms may be desirable because of their unique electronic and optical properties. At a fundamental level, the delicate balance of intermolecular forces of varied strengths, noncovalent interfacial interactions between molecular overlayers and supporting substrate surfaces, molecular organizations and crystallographic symmetry, the existence of polymorphs, kinetic vs thermodynamic control, and/or spatial confinement constraint has a critical impact on the crystallization pathway and growth of molecular thin films as well as polymorph selectivity. ${ }^{16-19}$ As a result of such complexity, it is often difficult to predict the structure of a molecular overlayer on a specific substrate, especially when the interfacial interaction is relatively weak and no significant guiding force such as chemisorption is directly involved. Rather unexpected results for physisorbed assemblies of small molecules have been discovered in recent years using structure-probing diffraction, ${ }^{20-24}$ scanning probe, ${ }^{25-27}$, and spectroscopic methods. ${ }^{28,29}$

Different types of substrate-induced ordering of molecular films have been reported in previous studies in the absence of prominent substrate-molecule interactions. Along the surface normal direction, terraced highly-oriented pyrolytic graphite (HOPG) has been shown to promote ordered stacking of a few small molecules via a lattice-matching template effect, where the step height of the HOPG substrate and the interlayer distance of a nucleating crystal plane can be related in a simple integer ratio. ${ }^{20,22-24}$ For in-plane ordering, ledge-directed epitaxy and 
commensurate/coincident epitaxy between a substrate and a molecular thin film were found to be responsible for a number of aligned crystal growths. For the former, a ledge joining a terrace plane and a step plane on a single-crystalline substrate surface may serve as the nucleation site if the dihedral angle of two crystal planes in a prenucleation aggregate resembles that of the substrate ledge, in addition to a lattice match between the supporting surface and the molecular overlayer. An example with a $1.0 \%$ lattice mismatch and a dihedral-angle difference of $0.6^{\circ}$ was given for the oriented nucleation and growth of benzoic acid on single-crystalline $\beta$-succinic acid. $^{30}$

As for the different modes of epitaxy, a classification scheme has been developed using a transformation matrix $C$ to relate the primitive lattice vectors of an overlayer with those of the substrate. ${ }^{16,31}$ Commensurate epitaxy, also called "point-on-point" coincidence where all the overlayer lattice points coinciding with the substrate's, may take place when all the matrix elements are an integer. However, such epitaxy is not often achievable considering the different symmetry and unit cell sizes of a molecular film compared to those of commonly used substrates. In contrast, the so-called "point-on-line" coincidence may be found when all the overlayer lattice points lie on one set of the substrate primitive lattice lines, which corresponds to two elements in one column of the matrix $C$ being integers. ${ }^{32}$ If only a fraction of the overlayer lattice points lies on the substrate primitive lattice lines with all matrix elements being a rational number, a large supercell consisting of several overlayer unit cells can be constructed such that its corner points are in registry with the substrate but those within do not. Another type of epitaxy showing "line-on-line" coincidence has also been found. ${ }^{33}$

With the understanding of the aforementioned different modes of epitaxy, organic surfaces of self-assembled monolayers (SAMs) and Langmuir-Blodgett (LB) films may provide 
a good platform for growth of ordered molecular films given the tuneability of surface functionalities and packing arrangements. Examples have been shown in previous studies for controlling polymorphism, ${ }^{34}$ morphology, ${ }^{35}$ and shape ${ }^{36}$ of biominerals, proteins, and inorganic and organic crystals. Crystal growths of calcium carbonate and barium sulfate beneath Langmuir monolayers of long-chain fatty acids have been studied in depth, where geometrical lattice matching, electrostatic binding, and stereochemical matching between a monolayer and a nucleating crystal plane were found to be responsible for preferential or selective ordering. ${ }^{37-40}$ Later, aligned growths of crystals have been further studied using ordered SAMs supported on metallic substrates to examine, e.g., an orientational match between the terminal carboxylic groups of the SAM and carbonate ions in the calcite plane ${ }^{41,42}$ as well as the impact of varying the lattice mismatch at the interface. ${ }^{36}$ However, understanding of epitaxial growth on organic surfaces is still limited. Besides, nearly all of the previous studies involved crystal growth in a solution environment, and the effect of solvent molecules or impurities on the crystallization process remains unclear.

Here, we report the crystal growths of four commonly used solvents-acetonitrile, ethanol, methanol, and water-on a hydrophobic, nonpolar SAM of n-alkanethiols at cryogenic temperatures using reflection high-energy electron diffraction (RHEED). Compared to previous studies on similar SAMs, only the small crystallizing molecules are present, and the interfacial overlayer structures and the potential template effects by a SAM can be examined in detail with controlled film thicknesses in an ultrahigh vacuum environment. Also, these four solvent molecules exhibit relatively stronger intermolecular interactions than the interfacial interaction per molecule, and thus preferential structural ordering guided by the SAM surface may not be obvious. However, it is somewhat unanticipated that acetonitrile and ethanol form a sustaining 
vertical order on a nonpolar SAM with long-range crystalline structures independent of the film thicknesses, unlike the randomly-oriented cubic ice crystallites formed of water. Coincident epitaxy with small lattice mismatches is found to be instrumental to these orderings, which are energetically favorable but without a dominant azimuthal orientation. In contrast, a preferred registry of methanol is found but only for a nominal thickness up to $\sim 1.2 \mathrm{~nm}$, with the unexpected observation of the high-temperature crystal structure. Such semi-commensurate epitaxy with a tensile lattice strain of nearly $7 \%$ becomes lost in films with thickness increase by even $<1 \mathrm{~nm}$. A phase change to the usual low-temperature one found at comparable temperatures is also observed. Our results encourage further investigations, both experimentally and theoretically, of the crystallization of molecular layers on ordered SAM surfaces with the help of epitaxial effects.

\section{EXPERIMENTAL SECTION}

Preparation of SAM Samples. Single-crystalline Au(111) substrates with a surface orientation accuracy of $<0.1^{\circ}$ were purchased from Princeton Scientific Corporation. Repeated rounds of argon-ion sputtering at $1.5 \mathrm{keV}$ at room temperature for $\sim 20$ minutes and annealing at 500$550^{\circ} \mathrm{C}$ for $20-30$ minutes at a base pressure of $<2 \times 10^{-8}$ torr were used for cleaning. The resulting atomically flat surfaces exhibited the characteristic streaky RHEED patterns ${ }^{43}$ and were also imaged using atomic force microscopy (AFM). Surface cleanliness without carbon or sulfur coverage was further confirmed with Auger electron spectroscopy. For the SAM fabrication, a standard procedure was followed. ${ }^{44}$ Briefly, a clean gold substrate was removed from vacuum and quickly immersed in a 2-mM 1-octadecanethiol (ODT, $98 \%$, Sigma Aldrich) in anhydrous ethanol for $48 \mathrm{~h}$. Afterward, the loose physisorbed thiol molecules were rinsed off by a copious 
amount of pure ethanol. Then the specimen was dried under ultrapure helium followed by its loading into vacuum on a sample plate with a K-type thermocouple directly attached to the specimen surface. Details of the RHEED apparatus may be found in previous reports. ${ }^{23,43}$ The sample stage integrated with a cryostat and a resistive heater was utilized to control the surface temperature in the range of $100-460 \mathrm{~K}$.

Depositions of Molecules and RHEED Experiments. Details of the effusion-style molecular doser assembly have been reported previously. ${ }^{23}$ Anhydrous acetonitrile (ACN, $99.8 \%$, with water $<50 \mathrm{ppm}$, Sigma Aldrich), ultrapure methanol (ACS spectrophotometric grade, $>99.9 \%$, Sigma Aldrich), anhydrous ethanol (99.8\%, Sigma Aldrich), and pure water (Milli-Q) were each loaded into a dedicated stainless steel reservoir and degassed via several freeze-pump-thaw cycles. Depositions of these molecules on SAMs were performed in an ultrahigh vacuum chamber with a base pressure of the order of $10^{-10}$ torr. The purity of the molecular sources and the absence of other atomic or molecular species (except for the residual hydrogen atoms and molecules) were confirmed using a residual gas analyzer. The SAM substrate was cooled to and maintained at $\sim 100 \mathrm{~K}$ for molecular depositions, using liquid nitrogen as a cryogen. The accuracy of the specimen temperature monitored by the K-type thermocouple in direct contact was $<1 \mathrm{~K}$. Nominal film thicknesses were obtained using the deposition times and rate of $\sim 1.0 \AA / \mathrm{s}$ calibrated by in-situ optical interferometry. Following the preparation, the structural changes in the deposited thin films on hydrophobic SAMs were monitored by RHEED during annealing to examine the temperature and film thickness dependences. ${ }^{23,24}$ The probe depth of $30 \mathrm{keV}$ electrons at a grazing incidence angle of $\theta_{\text {in }} \cong 1^{\circ}$ is $1-2 \mathrm{~nm}$. 


\section{RESULTS}
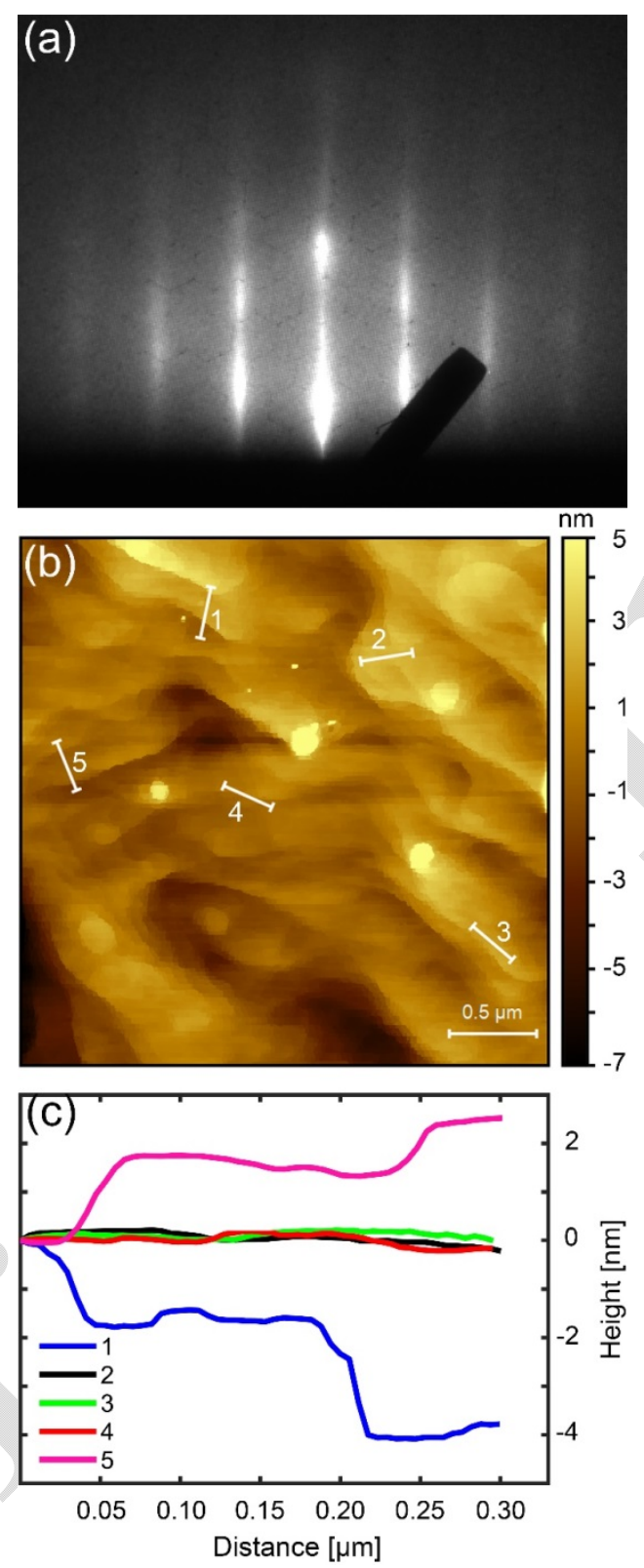

Figure 1. Diffraction of a SAM and surface topography of supporting Au(111). (a) RHEED pattern of an ODT SAM obtained with electrons propagating along $\langle 10\rangle$ of $\mathrm{Au}(111)$ at $\theta_{\text {in }}=1^{\circ}$ (b) AFM image of the single-crystalline Au(111) surface used for SAM depositions. (c) Selected line profiles as indicated in (b) showing an atomically smooth surface within few hundreds of nm but with topographical steps in a larger region. 


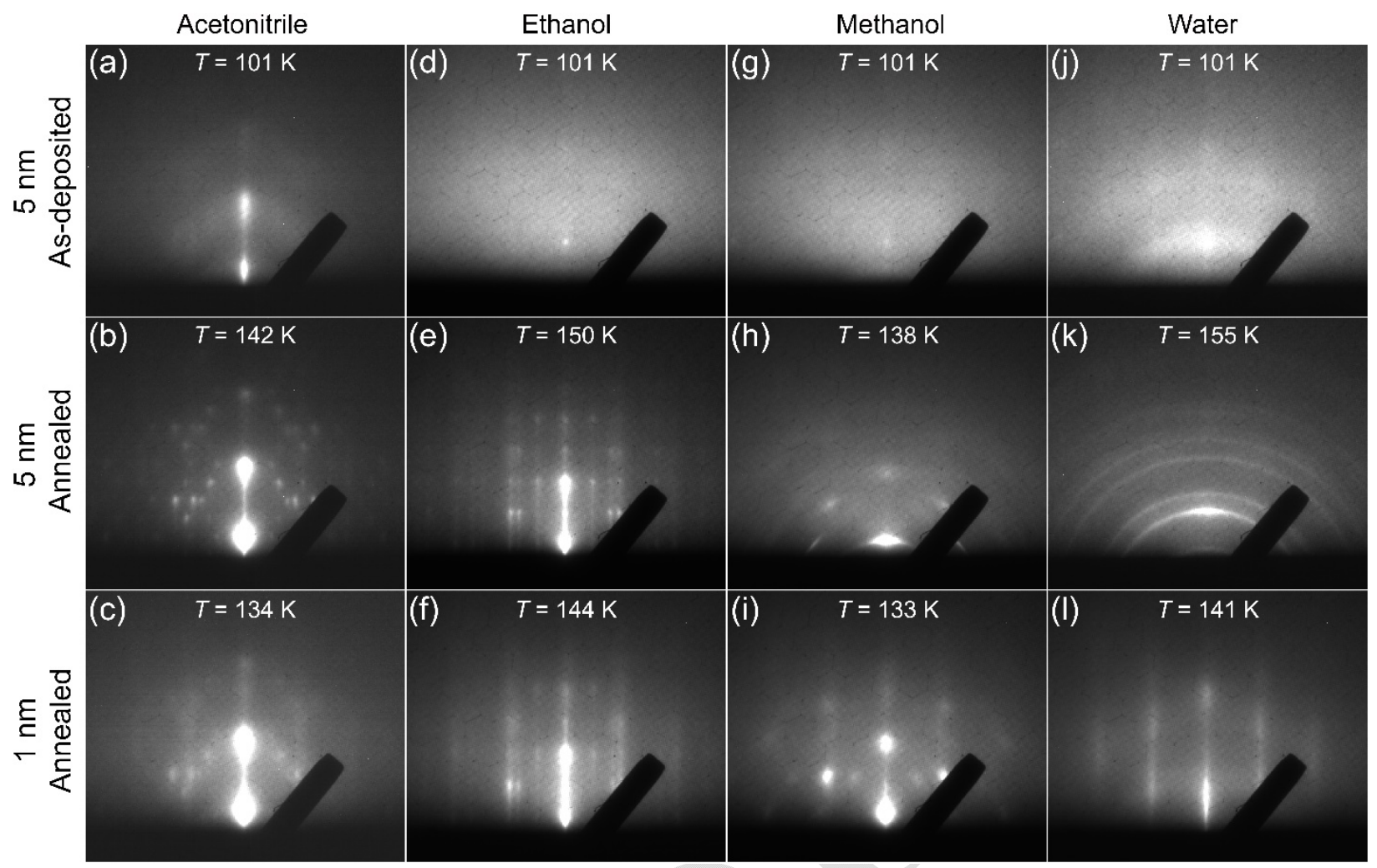

Figure 2. RHEED patterns of four different molecular films deposited on an ODT SAM at two different nominal film thicknesses ( $5 \mathrm{~nm}$ for top two rows and $1 \mathrm{~nm}$ for the bottom row). The top row is for the as-deposited films and the bottom two are for the annealed films.

A clear understanding of the SAM surface structure and topography is critical to overlayer studies. Shown in Figure 1 are a characteristic RHEED pattern of an as-deposited ODT SAM obtained at $100 \mathrm{~K}$, a typical AFM image of clean $\mathrm{Au}(111)$ acquired at room temperature, and the height profiles over a few selected lines in the micrograph. A detailed analysis of the RHEED patterns and the contributing SAM structures have been reported in a previous work. ${ }^{43}$ In short, the observation of intense Bragg spots arranged in a hut shape and overlapped with slanted diffuse diffraction bands indicates a major contribution by $\sim 10$-nm-sized ordered domains of the six-fold $(\sqrt{3} \times \sqrt{3}) R 30^{\circ}$ primary structure with tilted, "liquid-like" azimuthally-rotated thiolate molecules filling in between. The hexagonal lattice constant obtained is $a_{\mathrm{SAM}}=5.00 \AA$ for the well-packed regions, and the tilt angle for thiolate adsorbates is about $30^{\circ}$ from the surface 
normal direction. Furthermore, the clearly discernible vertical streaks in Figure 1a signify the presence of gold adatoms beneath the sulfur headgroups, which is consistent with the belief that these adatoms are induced during the SAM formation through relocating gold atoms from the $\mathrm{Au}(111)$ step edges, resulting in gold vacancy islands (pits) and consequently a locally stepped SAM surface with an average step height of $\sim 2.5 \AA$ often observed in STM studies. ${ }^{45,46}$ In addition, greater height differences as a result of the multiple interplanar distances of clean $\mathrm{Au}(111)$ (Figure 1, $\mathrm{b}$ and $\mathrm{c}$ ) are also present in the SAM terrace on the $\mu \mathrm{m}$ scale. Thus, an asdeposited SAM surface may seem far from an ideal one for epitaxial growths of molecular overlayers, given the different causes for its surface steps and topography and the limited size of the ordered domains.

Shown in Figure 2 are the RHEED patterns obtained from the four different molecular films at two different nominal thicknesses ( 1 and $5 \mathrm{~nm}$ ), at the deposition temperature of $101 \mathrm{~K}$ (top row) and after crystallization is mature at higher annealing temperatures (lower two rows); the same electron beam direction parallel to the $\langle 10\rangle$ of $\mathrm{Au}(111)$ was used for all these images. Significantly different patterns are found after annealing of the molecular thin films, while a mostly diffuse image is seen for each of the as-deposited films (with some diffraction spots observed in the center streak for ACN and two discernible broad diffraction bands for water), which indicates the extent of disorder in the immobilized molecular aggregates. It is apparent that at higher temperatures, water molecules form randomly oriented ice crystallites to give the Debye-Scherrer rings (Figure 2k), whereas the multiple Bragg spots observed for ACN (Figure 2, $\mathrm{b}$ and $\mathrm{c}$ ) and ethanol (Figure 2, e and $\mathrm{f}$ ) indicate the presence of certain surface ordering. A transition from an ordered film at $1 \mathrm{~nm}$ (Figure 2i) to the emergence of random orientation at 5 $\mathrm{nm}$ (Figure 2h) for methanol is certainly intriguing. These preliminary observations and 
comparisons hint at a story of rich phenomena for the growths of molecular overlayers on SAMs.

Ordered structures of ACN and coincident epitaxy. We first examine the overlayer structures of ACN, which have also been studied previously on $\mathrm{HOPG} .{ }^{24}$ Here, the observation of bright diffraction spots along the center streak and much fainter features on the sides (Figure 2a) indicates the clear presence of a stacking order along the surface normal direction in the asdeposited thin films at $101 \mathrm{~K}$, but the crystallites with extended in-plane horizontal orders are yet to grow. Apparently, certain interfacial influence is at work to form such a partial order instead of random aggregates serving as nuclei for the formation of randomly-oriented crystallites later. Upon thermal annealing of 5-nm films, a sharp spotty diffraction pattern is resulted (Figure 2b), which resembles that of the $\alpha$ phase observed on HOPG with a low step density (see below). Such a structural transformation signifies the growth of crystallites with extended 3-dimensional orders. Using the Scherrer formula we estimate that the in-plane average domain size reaches as large as $\sim 100 \mathrm{~nm}$, which is almost 10 times of that of the supporting $\mathrm{SAM}^{47} \mathrm{~A}$ similar arrangement of the RHEED pattern were also found from films as thin as $1 \mathrm{~nm}$ (Figure 2c) but with weaker diffraction intensities and larger spot widths over the SAM diffraction streaks, which is expected from reduced crystallite sizes and thicknesses.

An azimuthal rotation of the sample along the surface normal direction yields no significant changes in the diffraction pattern. Such an observation suggests that the crystal growth of ACN molecules does not adopt a unique in-plane orientation. Consistent with the simulation result using an azimuthal average (Figure 3a), thin-film ACN assemblies are found to crystallize into the high-temperature monoclinic $\alpha$ phase whose $\left[\overline{1} 0 \frac{1}{2}\right]$ is along the surface normal as the common stacking direction. ${ }^{24}$ Hence, the molecular axis of ACN molecules is parallel to the surface with each layer containing apparent dipole-dipole pairs. To understand 
such an orientational preference, we consider both the potential template effect supported by the SAM surface terrace ${ }^{22,24}$ and the possibility of some in-plane epitaxial relation between an ACN overlayer and the hydrophobic ODT SAM. Examination of the latter factor is suitable given the quasi-crystalline nature of a SAM with defined in-plane orientations with respect to the supporting $\mathrm{Au}(111)$ substrate.

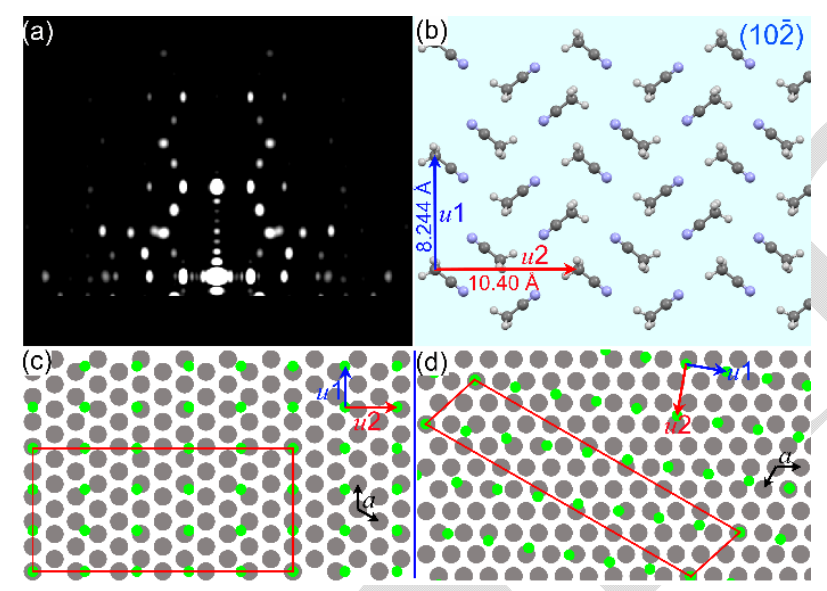

Figure 3. Coincident epitaxy of an ACN overlayer. (a) Simulated RHEED pattern of the

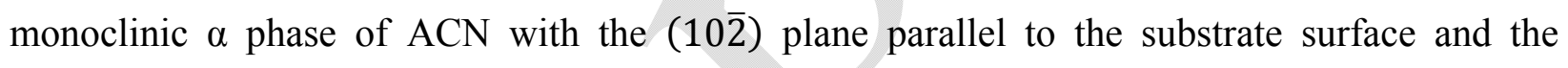
average of crystallite contributions over the entire azimuthal range. (b) Top-down view of the $(10 \overline{2})$ plane of the monoclinic $\alpha$ phase. The rectangular two-dimensional unit cell is denoted. (c) Schematic of an epitaxial relation between an ACN overlayer (green filled circles) and the hexagonal SAM surface (gray filled circles). The point-on-line supercell is denoted by the red rectangle. (d) Schematic of another epitaxial relation with a different supercell structure.

Shown in Figure $3 \mathrm{~b}$ is the $(10 \overline{2})$ plane of monoclinic ACN with the lattice constants of $u 1=8.244 \AA$ and $u 2=10.40 \AA$. Although the unit cells and lattice symmetry appear to be quite different between the ODT SAM and the ACN overlayer, these two structures are found to have multiple orientations of coincident matching on certain lattice points. For example, an overlap scheme is shown in Figure $3 \mathrm{c}$ where $u 1$ and $u 2$ of the ACN overlayer coincide with the $\langle 10\rangle$ and $\langle 11\rangle$ directions of the SAM, respectively. A large supercell indicated by the red rectangle can be 
identified where the corner lattice points of the overlayer coincide with those of the substrate. This supercell of coincident epitaxy can be described as $(5 \times 6 \sqrt{ } 3)$ with respect to the underlying SAM. Using the reported lattice constants, the theoretical lattice mismatches would be merely $-1.08 \%\left(3 * u 1=24.73 \AA\right.$ vs. $\left.5 * a_{\mathrm{SAM}}=25.0 \AA\right)$ and less than $+0.1 \%\left(5^{*} u 2=52.00 \AA\right.$ vs. $6 \sqrt{3} * a_{\mathrm{SAM}}=51.96 \AA$ ). Shown in Figure $3 \mathrm{~d}$ is another example of coincident epitaxy obtained by rotating the $\mathrm{ACN}$ overlayer by $10^{\circ}$ (with the overall pattern rotated by $90^{\circ}$ to maintain the panel aspect ratio), which would have theoretical lattice mismatches of $+0.3 \%$ and $-0.9 \%$ for the short and long cell axes, respectively. Experimentally, we do find an average tensile strain of $\sim 1.0 \%$ obtained from the horizontal positions of the $(1 \overline{1} \overline{4}),(1 \overline{2} \overline{4}),(1 \overline{2} \overline{3})$, and $(2 \overline{1} \overline{3})$ diffractions of $1-$ $\mathrm{nm}$ films as well as that of $1.3 \%$ compared to thicker films. This result agrees reasonably well with the picture that the interfacial overlayer adjusts slightly to better match with the substrate (hence to reduce the aforementioned larger negative mismatches) and this lattice strain dimishes to recover the strainless bulk for ACN layers away from the SAM surface. Thus, we find strong supports for coincident epitaxial matching as a major reason for specific ordering of $\mathrm{ACN}$ molecules on an ODT SAM.

Ordered structures of ethanol and coincident epitaxy. The amorphous-to-crystalline phase transition of interfacial ethanol is rich and also demonstrates results of a delicate balance between interfacial and intermolecular interactions. For this study, ethanol is found to exhibit a multi-step crystallization behavior. Initially, a strong center streak and two weak ones are observed in the temperature range of 107 to $117 \mathrm{~K}$ (Figure S1a). Above $117 \mathrm{~K}$, more diffraction streaks are visible and later at even higher temperatures the RHEED pattern becomes a combination of Bragg spots overlaying with sharper streaks (Figure S1, b and c). The diffraction pattern after the crystallization matures before the film desorption is shown in Figure 2e. Hence, 
the observation of streaks and overlaying spots later signifies the initial formation of first stacked two-dimensional (2D) networked layers without a vertical crystalline order, followed by the three-dimensional (3D) growth of crystallites at higher temperatures. This is reminiscent of the two-step amorphous-to-crystalline transition of interfacial methanol on hydrophobic smooth surfaces. ${ }^{23}$

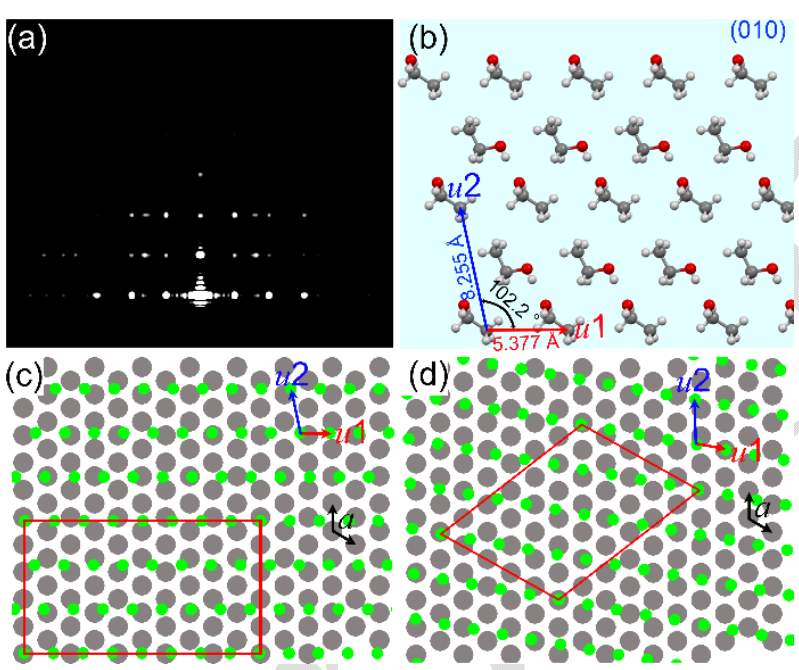

Figure 4. Coincident epitaxy of an ethanol overlayer. (a) Simulated RHEED pattern of the monoclinic $\alpha$ phase of ethanol with the (010) plane parallel to the substrate surface and the average of crystallite contributions over the entire azimuthal range. (b) Top-down view of the (010) plane of the monoclinic $\alpha$ phase. The two-dimensional unit cell is denoted by the parallelogram. (c) Schematic of an epitaxial relation between an ethanol layer (green filled circles) and the hexagonal SAM surface (gray filled circles). The point-on-line supercell is denoted by the red rectangle. (d) Schematic of another epitaxial relation with a different supercell structure.

However, the conversion of diffraction streaks into spots appears to be incomplete for ethanol even when the desorption begins. Thus, the remaining of streaks in Figure 2e indicates that the 2D-to-3D transition is not as complete as that of interfacial methanol. From the width of diffraction spots, we estimate the average 3D crystalline domain size to be about $120 \mathrm{~nm}$ using the Scherrer formula, ${ }^{47}$ which is similar to that of an $\mathrm{ACN}$ assembly. In comparison, the RHEED 
pattern of 1-nm films exhibits similar features except the observations of (i) more streak intensities relative to the spots' compared to those from 5-nm films, (ii) clearly broader vertical widths of the Bragg spots signifying a much reduced average thickness of 3D crystallites, and (iii) larger horizontal diffraction widths indicating a reduction in the average domain size. These results are expected for few-layered films. Furthermore, similar to the ACN case, no major changes are found in the patterns when different azimuthal angles of the sample are used for probing, which rules out the scenario of a unique in-plane preferential orientation.

In fact, coincident epitaxy is again found to support the growth of ordered ethanol overlayer structures on an ODT SAM. We use the lattice parameters of the monoclinic $\alpha$ phase of ethanol for kinematic scattering simulations, $a=5.377 \AA, b=6.882 \AA, c=8.255 \AA$, and $\beta=$ $102.2^{\circ}$. It is found that the $b$ axis is along to the surface normal direction, i.e., the (010) plane is parallel to the SAM surface. In such an orientation, the puckered hydrogen-bond (HB) chains extend along an in-plane direction sandwiched by the hydrophobic aliphatic part separated by van der Waals (vdW) interactions (Figure S2b), which also appears reasonable considering the layer's interaction with the hydrophobic ODT SAM. A satisfactory match can be seen between the Bragg spots (neglecting the remaining streak intensities) in the experimentally observed pattern (Figure 2e) and the simulated one from an azimuthally-rotated average with the common $b$ axis (Figure 4a). Shown in Figure $4 \mathrm{~b}$ is the top-down view of the (010) plane, where the 2dimensional unit cell can be identified as a parallelogram with the lattice constants of $u 1=5.377$ $\AA$ and $u 2=8.255 \AA$ separated by the angle of $102.2^{\circ}$. Even though the lattice constants and symmetry appear to be different from those of the supporting SAM, two examples of the many coincident epitaxial matchings are given in Figure 4, c and d. In the first orientation, the $u 1$ and [13] axes of ethanol(010) are largely parallel to the $\langle 11\rangle$ and $\langle 10\rangle$ directions of the ODT SAM. 
The matching supercell, termed as $(5 \sqrt{3} \times 5)$ relative to the SAM structure, may have lattice mismatches of $0.656 \%\left(8^{*} u 1=43.02 \AA\right.$ vs. $\left.5 \sqrt{ } 3^{*} a_{\mathrm{SAM}}=43.3 \AA\right)$ and $3.16 \%([13]=24.21 \AA$ vs. $5^{*} a_{\mathrm{SAM}}=25.0 \AA$ ). Experimentally, a lattice expansion of $3.3 \%$ compared to the bulk value is obtained from fits of the horizontal positions of the (031) and (032) diffractions from 1-nm films, which signifies the interfacial effect along the $u 2$ (closer to [13]) axis. Hence, it can be concluded that the first few hydrogen-bonded ethanol layers are more strained to conform with the substrate lattice but the strain is relieved in layers away from the surface to recover the bulk lattice. In another example, coincident epitaxial matching with limited lattice mismatches of $-0.9 \%$ and $-1.5 \%$ can be seen from the ethanol overlayer rotated by $10^{\circ}$ (Figure $4 \mathrm{~d}$ ). These overlayer findings resemble those of $\mathrm{ACN}$, which reasonably explain the similarity between the ACN and ethanol results.

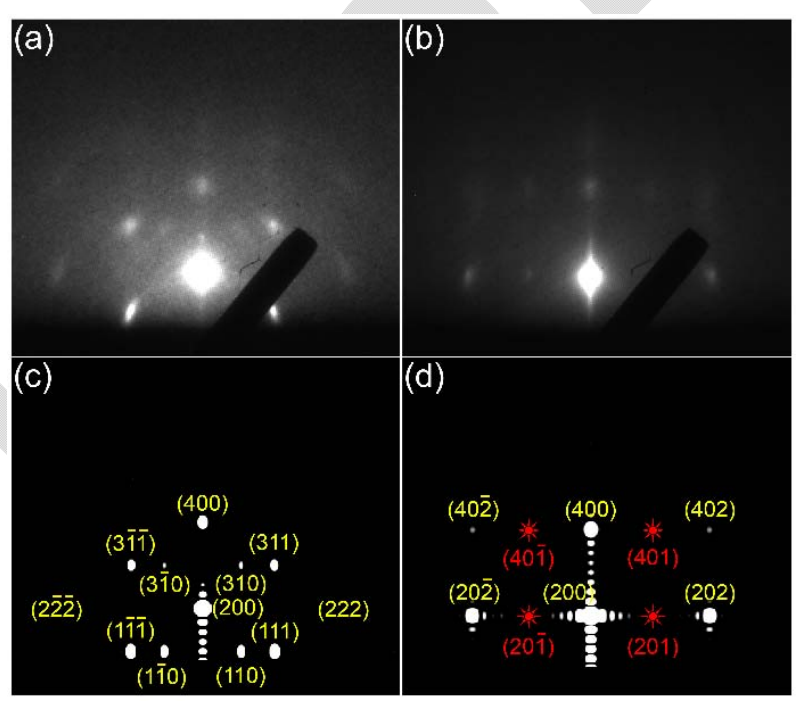

Figure 5 RHEED patterns of 1.2-nm methanol films acquired with the electron beam parallel to (a) the $\langle 10\rangle$ direction and (b) the $\langle 11\rangle$ direction of $\mathrm{Au}(111)$. (c-d) Corresponding simulated patterns calculated considering a sixfold average of the methanol $\beta$ phase with the (100) plane parallel to the substrate surface. The red stars in (d) denote the theoretically absent diffractions that are still observed with weak intensities. Their appearance implies a symmetry breaking along the HB chains originally in equal spacings (see text). 


\section{Semi-commensurate epitaxy of monolayer methanol and quick loss of interfacial}

ordering. Distinct differences are observed in the amorphous-to-crystalline transition and overlayer structures of methanol on an ODT SAM. At a first glance, annealing of 5-nm methanol films yields weak diffraction rings overlapped with a few curved diffraction spots, which signifies the formation of randomly oriented crystallites with preference for a certain orientation (Figure 2h). However, further thickness-dependent experiments in the range of 0.2 to $15 \mathrm{~nm}$ show that the diffraction rings begin to appear for nominal thicknesses above $1 \mathrm{~nm}$ whereas the diffraction spots completely disappear when the thickness reaches $15 \mathrm{~nm}$ (Figure S3). At $1 \mathrm{~nm}$ and below, only several Bragg spots are observed overlaying with the weakened SAM diffraction streaks (Figure 2i), in a different arrangement than in Figure 2h. More intriguingly, the RHEED pattern depends on the incident azimuth used. A different spotty pattern is found when the sample is azimuthally rotatedby $30^{\circ}$ and probed, which indicates a unique crystalline order relative to the supporting SAM (Figure 5, a and b). These observations suggest the significance of a substrate-induced ordering in both in-plane and out-of-plane directions at the interface but also its limited range. In comparison, the coincident epitaxial growth with a vertical stacking order (i.e., a common vertical axis) is still seen for thicker ACN and ethanol films with more than $15 \mathrm{~nm}$.

Both orthorhombic low-temperature $\alpha$ phase $(a=4.6469 \AA, b=4.9285 \AA, c=9.0403 \AA)$ and high-temperature $\beta$ phase $(a=6.401 \AA, b=7.220 \AA$, and $c=4.6470 \AA)$ are considered for kinematic scattering simulations. ${ }^{48}$ To our surprise, an ultrathin methanol overlayer does not crystallize into the $\alpha$ phase commonly seen in vapor-deposited films at cryogenic temperatures, as no acceptable theoretical agreement is found. Instead, consistent patterns with the indicies of the observed Bragg spots are obtained using the $\beta$ phase, with the (100) plane parallel to the 

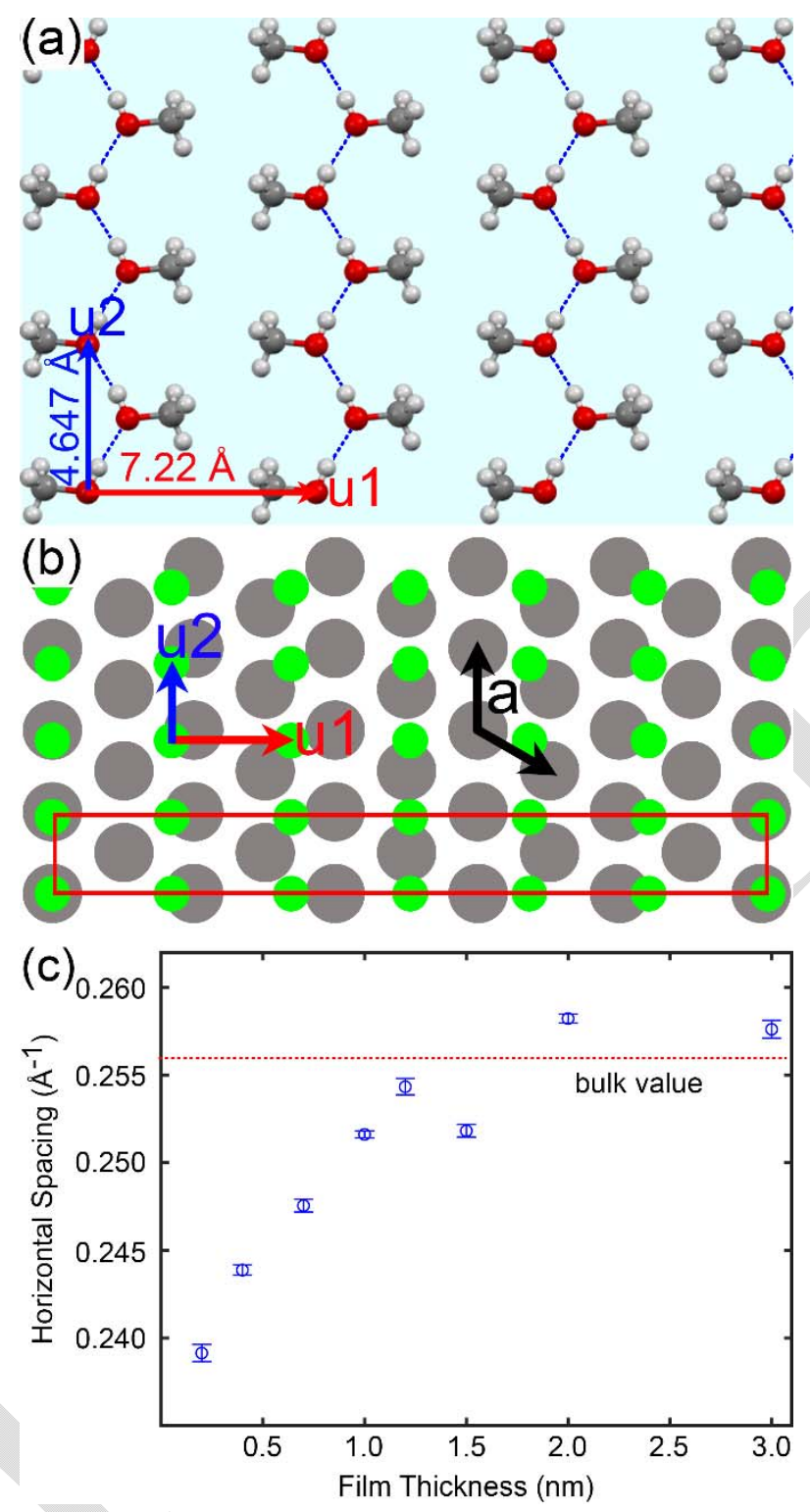

Figure 6. Commensurate epitaxy of an methanol overlayer. (a) Top-down view of the (100) plane (in direct contact with the SAM) of the monoclinic $\beta$ phase of methanol. The primitive lattice vectors are denoted. (b) Schematic of possible commensurate (black rectangle) and coincident (red rectangle) epitaxial relations between a methanol overlayer (green filled circles, without lattice strain) and the SAM substrate (gray filled circles). (c) Dependence of the horizontal diffraction spacing on the apparent film thickness, which shows the amount of lattice strain in ultrathin methanol. The red dashed line indicates the value derived from the bulk lattice constants. The small error bars are related to the uncertainties from fits of the diffraction spot positions; a larger uncertainty of up to $2 \%$ may come from the camera length due to the grazing incidence of RHEED. 
substrate surface and a sum of sixfold $\left(60^{\circ}\right)$ azimuthal rotations given the supporting SAM structure (Figure 5, c and d). In such a configuration, the HB chains extend along the sixfold $\langle 10\rangle$ directions of the ODT SAM with a small upward or downward molecular tilt of $12^{\circ}$. The top-down view of the unstrained (100) plane and its lattice points relative to those of the SAM are shown in Figure 6, a and b. The 2D unit cell is rectangular in shape with the lattice constants of $u 1=7.220 \AA$ and $u 2=4.647 \AA$.

Following the analyses for ACN and ethanol overlayers, an initial look of the two overlaying structures in Figure $6 \mathrm{~b}$ gives the hint for a possible supercell indicated by the red rectangle; $u 1$ and $u 2$ of methanol(100) are parallel to the $\langle 10\rangle$ and $\langle 11\rangle$ directions of the ODT SAM. However, a relative large deviation is found in the coincidence of the corner lattice points of the $(5 \sqrt{3} \times 1)$ supercell if the methanol overlayer remains unstrained, with theoretical lattice mismatches of $0.046 \%\left(6 * u 1=43.32 \AA\right.$ vs. $\left.5 \sqrt{ } 3 * a_{\mathrm{SAM}}=43.3 \AA\right)$ and $7.06 \%(u 2=4.6470 \AA$ vs. $a_{\mathrm{SAM}}=5.00 \AA$ ). More intriguingly, unlike the $\mathrm{ACN}$ and ethanol results, an ultrathin methanol overlayer is experimentally observed to adopt one particular azimuthal orientation with respect to the supporting ODT SAM, even though different coincidences are found to exist at other azimuths theoretically. This distinct contrast implies that coincident epitaxy may not work in the same fashion for the oriented growth of ultrathin methanol as for that of ACN and ethanol overlayers. Such a realization also provides clues for the quick loss of ordering in slightly thicker methanol films, which is again contrary to the $\mathrm{ACN}$ and ethanol results.

A further analysis of the experimental patterns shows that the horizontal spacing between the (311) Bragg spot and the center streak deviates from the theoretical value of $\sqrt{u 1^{* 2}+u 2^{* 2}}=$ $0.2559 \AA^{-1}$. The dependence of this (11) horizontal spacing on the nominal film thickness is plotted in Figure 6c. For the thinnest film studied, which has a partial surface coverage, the (11) 
horizontal spacing exhibits a significant $6.6 \%$ reduction in reciprocal space compared to the bulk value; in contrast, the (10) horizontal spacing has little deviation. Thus, the $6.6 \%$ reciprocal contraction is mainly contributed by the $u 2^{*}$ direction, which corresponds to a large lattice expansion along the hydrogen-bonded chains. The agreement between the experiment and the aforementioned methanol(100)-SAM structural comparison is remarkable, whose implications will be discussed below. However, such a lattice expansion in a "semi-commensurate" overlayer-a commensurate $1: 1$ match along $u 2$ and a near-perfect supercell along $u 1$-quickly diminishes as the film thickness increases slightly, and the strainless bulk value is reached at around $1.2 \mathrm{~nm}$. Furthermore, the appearance of less-defined, curved diffractions and DebyeScherrer rings from thicker films indicates a change in the crystallinity and vertical ordering in contrast to the ACN and ethanol results (Figures $2 \mathrm{~h}$ and S3). In fact, the ring pattern obtained from 15 -nm films agrees better with the low-temperature $\alpha$-phase structure, not the $\beta$-phase one (Figure S3j). Such an observation signifies a return from an interface-dominant scenario to the common bulk governed by the intermolecular forces.

\section{DISCUSSION}

The rich overlayer structures observed in this study form a challenge to the understanding of ordering of simple molecules on a hydrophobic SAM. Given no significant interfacial interactions such as chemisorption, Coulombic forces, HBs, or steric matching with the SAM surface, a molecular overlayer formed of randomly-oriented crystallites would be anticipated, which is indeed the case for cubic ice of polar and HB-forming water molecules but apparently not so for the other three solvent molecules used, even as they also exhibit similar intermolecular interactions. Highly polar ACN and HB-forming methanol may be considered resembling from 
the molecular structure viewpoint with different functional groups. In terms of assembly structures, both methanol and ethanol form extended HB chains in their solid states. However, experimental results show that comparable epitaxial and vertical-ordering results are found in ACN and ethanol thin films, whereas a highly-ordered methanol overlayer only exists at the interface and loses its orientational order away from the SAM surface. The characterization scheme for the different modes of epitaxy provides some help to make sense of the results, especially regarding the consistence between the anticipated and observed lattice strains near the SAM and the relief in thicker films. However, the fundamental reasons for the formation of these ordered overlayers are not fully understood.

It is noted that the principal molecular axes in crystalline $\alpha$ - $\operatorname{CN}(10 \overline{2}), \alpha$-ethanol $(010)$, and $\beta$-methanol(100) are either parallel or essentially parallel to the surface (Figures $3 \mathrm{~b}$, $4 \mathrm{~b}$, and 6a). Incidentally, these crystal planes also contain the most important intermolecular interactions for these molecules, with hydrophobic $\mathrm{C}-\mathrm{H}$ bonds of the methyl or ethyl groups pointing toward out-of-plane directions (Figure S2). As such, the orientations of the crystallized films fit the chemical intuition about the preference of hydrophobic groups being closer and interacting with the hydrophobic SAM surface while the HB or dipole-dipole interactions between neighboring molecules remain intact and minimally perturbed. At these SAM-molecule interfaces, the energy may be further lowered via optimizations of the interfacial vdW interactions by allowing low percentage of structural strains to achieve better lattice matches, as shown earlier in the coincident epitaxies for $\alpha-\operatorname{ACN}(10 \overline{2})$ and $\alpha$-ethanol(010). However, given no specific azimuth of the epitaxy is dominant and also the much larger domain size by $\sim 1$ order of magnitude compared to that of the underlying SAM, we argue that the energy landscape as a function of the in-plane azimuthal angle must contain many local minima corresponding to the large number of 
possible coincident epitaxial matches, but the energy barriers should be limited so that the joining and growth of crystallite domains into enlarged ones can still be achieved.

Along the surface normal direction, stacking of $\alpha-\operatorname{ACN}(10 \overline{2})$ and $\alpha$-ethanol(010) layers spaced by vdW hydrophobic groups remains preferred. A better 3D crystalline order is certainly found in ACN thin films based on the sharp Bragg spots in Figure 2b, whereas the azimuthal match between ethanol layers appears to be less defined and extended according to the still streak-looking diffractions (and therefore more 2D-layered nature) in Figure 2e. This difference may be due to the more apparent separation between adjacent ethanol bilayer sheets by the hydrophobic ethyl groups (Figure S2b). In addition, the surface topography of an ODT SAM (Figure 1, b and c) may also play the role of a template to a certain extent to allow crystalline sheets grown on neighboring terrace steps to join together. This could be particularly suitable for the case of ethanol where the vertical lattice constant $b=6.882 \AA$ is close to 3 times of the interplanar distance of $\mathrm{Au}(111)$ within a mismatch of $2.57 \%$. For $\mathrm{ACN}$, its complex crystallization kinetics is recently examined and noted, ${ }^{49}$ and we also observe the existence of some vertical ordering during initial deposition on an ODT SAM at $\sim 100 \mathrm{~K}$ before notable epitaxial matches become more mature by annealing (Figure 2a). Possible contributing factors include a preference of nucleus-forming, in-plane dimer configurations due to dipole interactions as well as the topographical template effect from the SAM for stacking. ${ }^{24}$

The unexpected methanol overlayer results provide rich information about crystallization on surfaces like an ODT SAM. It is certainly unique to see the choice of a specific azimuth of $\beta$ methanol(100), not even in the supposed $\alpha$ phase, for a semi-commensurate epitaxy with however the trade-off of a large lattice strain. According to an $a b$ initio theoretical study, the cohesive energy per methanol molecule in the $\alpha$ phase at zero pressure is lower by approximately 
$20 \mathrm{meV}$, i.e. less than $5 \%$ of the overall, than that in the $\beta$ phase. ${ }^{50}$ Thus, it could be possible that an energetically-favored epitaxy such as commensuration and/or ultralow lattice mismatch causes the interfacial structural change. In this study, the stabilization by the specific semicommensurate epitaxy of $\beta$-methanol(100) should be appreciably more than that provided by the probable coincident epitaxial scenario as seen in $\mathrm{ACN}$ and ethanol overlayers, even with the penalty from the accompanied lattice expansion of $6.6 \%$ experimentally found along the HB direction. Hence, the general idea of the preference of more commensurate epitaxy over coincident match in an overlayer appears to apply here, although details need to be taken into account. Furthermore, since HBs are more flexible than covalent ones, methanol molecules may be able to adjust their intracell positions to compensate for the imposed energy cost. In fact, the observation of nonzero intensities for the theoretically forbidden diffraction spots in Figure $5 \mathrm{~b}$ signifies a symmetry breaking along the HB direction, likely producing unequal and alternate $\mathrm{HB}$ distances between neighboring molecules in a chain.

However, the aforementioned picture does not fully explain why $\alpha$-methanol(001) was not the overlayer structure when a semi-commensurate epitaxy could also be induced in a comparable fashion: theoretical lattice mismatches of $7.06 \%$ along the HB direction ( $a=4.6469$ $\AA$ vs. $\left.a_{\mathrm{SAM}}=5.00 \AA\right)$ and $0.406 \%$ in the perpendicular chain-to-chain direction $(7 * b=34.4995$ $\AA$ vs. $4 \sqrt{ } 3 * a_{\text {SAM }}=34.64 \AA$ ). A main difference is that the zigzag HBs are much out-of-plane in the $\alpha$ phase unlike those essentially in the plane in the $\beta$ phase, although still reasonably spaced by hydrophobic methyl groups (Figure S4). A theoretical comparison for this SAM-methanol overlayer system with different lattice structures may provide further insights, although carefully tuned force-field parameters that can reproduce the experimental results are needed. One possible reason for the preference of the $\beta$ phase over the $\alpha$ one might be due to the dihedral 
angles of the methyl groups, where $\alpha$-methanol(001) appears to have a higher chance to encounter more steric interference due to twice as many $\mathrm{C}-\mathrm{H}$ bonds pointing toward the SAM that is also terminated by methyl groups.

However, the epitaxial match of interfacial $\beta$-methanol(100) poses very limited guidance for the crystallization of upper layers. Such a result well demonstrates the delicate balance between interfacial and intermolecular interactions. First, the relief of the lattice strain is found to be complete in $\sim 4$ layers nominally $(1.2 \mathrm{~nm}$ divided by the interlayer distance of a/2 = 3.201 $\AA$ ). Second, the tendency to resume the low-temperature $\alpha$-phase structure becomes high when the nominal film thickness increases by just a few nm, which implies a lesser role for ordering by the largely vdW interactions between these methanol layers. As a result, the orientation and phase mismatches cause methanol molecules to crystallize in random directions, leading to the result of diffraction rings that appears to be similar to that of water but in a superficial way.

For water, multiple factors may prevent an ordered growth on a hydrophobic SAM. In the cubic ice structure $(a=6.358 \AA)$, the tetrahedrally connected HBs form a 3D network without obvious cleavage planes. Considering the (111) plane with a lower density of HBs to break, an in-plane lattice mismatch of $10.1 \%$ is present, which makes a uniform $2 \mathrm{D}$ stretch of HBs for a commensurate epitaxy unlikely. Coincident epitaxy might be possible given the same hexagonal in-plane symmetry with, e.g., $10 * a=44.958 \AA$ matching $9 * a_{\mathrm{SAM}}=45.00 \AA$. However, crystallites of water cubic ice tend to be small in size presumably owing to a higher nucleus density as a result of strong intermolecular HBs in no preference of certain orientations. This result shows again that both possible epitaxial relations and crystallization kinetics, which fundamentally arise from interfacial and intermolecular forces, respectively, should be taken into consideration for potential engineering of ordered crystal growths on surfaces. 


\section{CONCLUSION}

In this study, we report the crystallization of four common solvent molecules at cryogenic temperatures on a hydrophobic SAM substrate using reflection high-energy electron diffraction. Despite the absence of significant interactions between the supporting surface and deposited molecules, acetonitrile, ethanol, and ultrathin methanol crystallize with a common crystallographic axis along the surface normal direction. Closer inspections of the crystal structures and their orientations with respect to the ODT SAM reveal that the oriented growths are favored because of the presence of in-plane epitaxy. More specifically, ordered structures of acetonitrile and ethanol assemblies are facilitated by a large number of in-plane coincident epitaxial matches where the overlayer lattice points coincide with those of the substrate by coincidence with low percentage of lattice strains. In contrast, a specific semi-commensurate epitaxial relation is achieved in ultrathin methanol overlayers by stretching and adjusting the HB chains, which leads to the oriented growth in both in-plane and out-of-plane directions but the ordering is quickly lost in slightly thicker films. Based on these experimental findings, we discuss the energetics for the crystal growths and demonstrate the underlying delicate balance between interfacial and intermolecular interactions. Our study shows that SAM surfaces with different functional tail groups and in-plane lattice structures may be used as templates to design oriented growths of crystals with implications in, e.g., molecular electronics, biotechnology, and liquid crystal displays.

\section{- Supporting Information}

The Supporting Information is available free of charge.

RHEED images of ethanol thin films in different temperature ranges, side view of the crystal structures of ACN, ethanol, and methanol, RHEED images of methanol thin films in 
different thicknesses, and side view of the two phases of solid methanol

\section{- AUTHOR INFORMATION}

\section{Corresponding Author}

*E-mail: yang@uh.edu Phone:+1 713-743-6022.

\section{ORCID}

Ding-Shyue Yang: 0000-0003-2713-9128

\section{Notes}

The authors declare no competing financial interests.

\section{- ACKNOWLEDGMENT}

This research was supported by a National Science Foundation CAREER Award (Grant no.

CHE-1653903) and the R. A. Welch Foundation (Grant no. E-1860).

\section{REFERENCES}

(1) Kobayashi, S.; Nishikawa, T.; Takenobu, T.; Mori, S.; Shimoda, T.; Mitani, T.; Shimotani, H.; Yoshimoto, N.; Ogawa, S.; Iwasa, Y. Control of Carrier Density by SelfAssembled Monolayers in Organic Field-Effect Transistors. Nat. Mater. 2004, 3, $317-$ 322.

(2) Klauk, H.; Zschieschang, U.; Pflaum, J.; Halik, M. Ultralow-Power Organic Complementary Circuits. Nature 2007, 445, 745-748.

(3) Sekitani, T.; Yokota, T.; Zschieschang, U.; Klauk, H.; Bauer, S.; Takeuchi, K.; Takamiya, M.; Sakurai, T.; Someya, T. Organic Nonvolatile Memory Transistors for Flexible Sensor Arrays. Science (80-. ). 2009, 326, 1516-1519.

(4) Stöhr, J.; Samant, M. G.; Lüning, J.; Callegari, A. C.; Chaudhari, P.; Doyle, J. P.; Lacey, J. A.; Lien, S. A.; Purushothaman, S.; Speidell, J. L. Liquid Crystal Alignment on Carbonaceous Surfaces with Orientational Order. Science (80-. ). 2001, 292, 2299-2302.

(5) Kim, J. H.; Yoneya, M.; Yokoyama, H. Tristable Nematic Liquid-Crystal Device Using Micropatterned Surface Alignment. Nature 2002, 420, 159-162.

(6) Kumar, S.; Kim, J. H.; Shi, Y. What Aligns Liquid Crystals on Solid Substrates? The Role 
of Surface Roughness Anisotropy. Phys. Rev. Lett. 2005, 94, 077803.

(7) Pisula, W.; Zorn, M.; Chang, J. Y.; Müllen, K.; Zentel, R. Liquid Crystalline Ordering and Charge Transport In Semiconducting Materials. Macromol. Rapid Commun. 2009, 30, 1179-1202.

(8) Dinelli, F.; Murgia, M.; Levy, P.; Cavallini, M.; Biscarini, F.; De Leeuw, D. M. Spatially Correlated Charge Transport in Organic Thin Film Transistors. Phys. Rev. Lett. 2004, 92, 116802 .

(9) Wang, L.; King, I.; Chen, P.; Bates, M.; Lunt, R. R. Epitaxial and Quasiepitaxial Growth of Halide Perovskites: New Routes to High End Optoelectronics. APL Materials. American Institute of Physics Inc. October 1, 2020, p 100904.

(10) Ban, C.; George, S. M. Molecular Layer Deposition for Surface Modification of LithiumIon Battery Electrodes. Advanced Materials Interfaces. Wiley-VCH Verlag November 7, 2016, p 1600762.

(11) Eremtchenko, M.; Schaefer, J. A.; Tautz, F. S. Understanding and Tuning the Epitaxy of Large Aromatic Adsorbates by Molecular Design. Nature 2003, 425, 602-605.

(12) Yokoyama, D. Molecular Orientation in Small-Molecule Organic Light-Emitting Diodes. J. Mater. Chem. 2011, 21, 19187-19202.

(13) Tonnelé, C.; Stroet, M.; Caron, B.; Clulow, A. J.; Nagiri, R. C. R.; Malde, A. K.; Burn, P. L.; Gentle, I. R.; Mark, A. E.; Powell, B. J. Elucidating the Spatial Arrangement of Emitter Molecules in Organic Light-Emitting Diode Films. Angew. Chemie - Int. Ed. 2017, 56, 8402-8406.

(14) Liu, F.; Zhang, Z.; Rong, X.; Yu, Y.; Wang, T.; Sheng, B.; Wei, J.; Zhou, S.; Yang, X.; Xu, F.; Qin, Z.; Zhang, Y.; Liu, K.; Shen, B.; Wang, X. Graphene-Assisted Epitaxy of Nitrogen Lattice Polarity GaN Films on Non-Polar Sapphire Substrates for Green Light Emitting Diodes. Adv. Funct. Mater. 2020, 30, 2001283.

(15) Horowitz, G. Evidence for N-Type Conduction in a Perylene Tetracarboxylic Diimide Derivative. Adv. Mater. 1996, 8, 242-245.

(16) Hooks, D. E.; Fritz, T.; Ward, M. D. Epitaxy and Molecular Organization on Solid Substrates. Adv. Mater. 2001, 13, 227-241.

(17) Carter, P. W.; Ward, M. D. Topographically Directed Nucleation of Organic Crystals on Molecular Single-Crystal Substrates. J. Am. Chem. Soc. 1993, 115, 11521-11535.

(18) Jones, A. O. F.; Chattopadhyay, B.; Geerts, Y. H.; Resel, R. Substrate-Induced and ThinFilm Phases: Polymorphism of Organic Materials on Surfaces. Advanced Functional Materials. Wiley-VCH Verlag April 21, 2016, pp 2233-2255.

(19) Hamilton, B. D.; Ha, J. M.; Hillmyer, M. A.; Ward, M. D. Manipulating Crystal Growth and Polymorphism by Confinement in Nanoscale Crystallization Chambers. Acc. Chem. Res. 2012, 45, 414-423.

(20) Yang, D. S.; Zewail, A. H. Ordered Water Structure at Hydrophobic Graphite Interfaces 
Observed by 4D, Ultrafast Electron Crystallography. Proc. Natl. Acad. Sci. U. S. A. 2009, 106, 4122-4126.

(21) Souda, R.; Aizawa, T. Crystallization Kinetics of Water on Graphite. Phys. Chem. Chem. Phys. 2018, 20, 21856-21863.

(22) He, X.; Wu, C.; Rajagopal, K.; Punpongjareorn, N.; Yang, D. S. Ordered Ionic Liquid Structure Observed at Terraced Graphite Interfaces. Phys. Chem. Chem. Phys. 2016, 18, 3392-3396.

(23) He, X.; Wu, C.; Yang, D. S. Communication: No Guidance Needed: Ordered Structures and Transformations of Thin Methanol Ice on Hydrophobic Surfaces. J. Chem. Phys. 2016, 145.

(24) Wu, C.; Yang, D. S. Ordered Structures and Morphology-Induced Phase Transitions at Graphite-Acetonitrile Interfaces. J. Phys. Chem. C 2019, 123, 22390-22396.

(25) Lawton, T. J.; Carrasco, J.; Baber, A. E.; Michaelides, A.; Sykes, E. C. H. HydrogenBonded Assembly of Methanol on Cu(111). Phys. Chem. Chem. Phys. 2012, 14, 1184611852.

(26) Liriano, M. L.; Larson, A. M.; Gattinoni, C.; Carrasco, J.; Baber, A. E.; Lewis, E. A.; Murphy, C. J.; Lawton, T. J.; Marcinkowski, M. D.; Therrien, A. J.; Michaelides, A.; Sykes, E. C. H. Chirality at Two-Dimensional Surfaces: A Perspective from Small Molecule Alcohol Assembly on Au(111). J. Chem. Phys. 2018, 149, 034703.

(27) Clair, S.; Pons, S.; Seitsonen, A. P.; Brune, H.; Kern, K.; Barth, J. V. STM Study of Terephthalic Acid Self-Assembly on Au(111): Hydrogen-Bonded Sheets on an Inhomogeneous Substrate. J. Phys. Chem. B 2004, 108, 14585-14590.

(28) Tylinski, M.; Smith, R. S.; Kay, B. D. Structure and Desorption Kinetics of Acetonitrile Thin Films on Pt(111) and on Graphene on Pt(111). J. Phys. Chem. C 2020, 124, 25212530 .

(29) Therrien, A. J.; Hensley, A. J. R.; Hannagan, R. T.; Schilling, A. C.; Marcinkowski, M. D.; Larson, A. M.; McEwen, J. S.; Sykes, E. C. H. Surface-Templated Assembly of Molecular Methanol on the Thin Film "29” Cu(111) Surface Oxide. J. Phys. Chem. C 2019, 123, 2911-2921.

(30) Carter, P. W.; Ward, M. D. Topographically Directed Nucleation of Organic Crystals on Molecular Single-Crystal Substrates. J. Am. Chem. Soc. 1993, 115, 11521-11535.

(31) Hoshino, A.; Isoda, S.; Kurata, H.; Kobayashi, T. Scanning Tunneling Microscope Contrast of Perylene-3,4,9,10- Tetracarboxylic-Dianhydride on Graphite and Its Application to the Study of Epitaxy. J. Appl. Phys. 1994, 76, 4113-4120.

(32) Hoshino, A.; Isoda, S.; Kurata, H.; Kobayashi, T. Scanning Tunneling Microscope Contrast of Perylene-3,4,9,10- Tetracarboxylic-Dianhydride on Graphite and Its Application to the Study of Epitaxy. J. Appl. Phys. 1994, 76, 4113-4120.

(33) Mannsfeld, S. C. B.; Leo, K.; Fritz, T. Line-on-Line Coincidence: A New Type of Epitaxy Found in Organic-Organic Heterolayers. Phys. Rev. Lett. 2005, 94. 
(34) Hiremath, R.; Basile, J. A.; Varney, S. W.; Swift, J. A. Controlling Molecular Crystal Polymorphism with Self-Assembled Monolayer Templates. J. Am. Chem. Soc. 2005, 127, $18321-18327$.

(35) Lee, A. Y.; Ulman, A.; Myerson, A. S. Crystallization of Amino Acids on Self-Assembled Monolayers of Rigid Thiols on Gold. Langmuir 2002, 18, 5886-5898.

(36) Pokroy, B.; Aizenberg, J. Calcite Shape Modulation through the Lattice Mismatch between the Self-Assembled Monolayer Template and the Nucleated Crystal Face. CrystEngComm 2007, 9, 1219-1225.

(37) Mann, S.; Heywood, B. R.; Rajam, S.; Birchall, J. D. Controlled Crystallization of CaCO3 under Stearic Acid Monolayers. Nature 1988, 334, 692-695.

(38) Heywood, B. R.; Mann, S. Organic Template-Directed Inorganic Crystallization: Oriented Nucleation of BaSO4 under Compressed Langmuir Monolayers. J. Am. Chem. Soc. 1992, 114, 4681-4686.

(39) Heywood, B. R.; Mann, S. Template-Directed Inorganic Crystallization: Oriented Nucleation of Barium Sulfate under Langmuir Monolayers of an Aliphatic Long Chain Phosphonate. Langmuir 1992, 8, 1492-1498.

(40) Heywood, B. R.; Mann, S. Template-directed Nucleation and Growth of Inorganic Materials. Adv. Mater. 1994, 6, 9-20.

(41) Aizenberg, J.; Black, A. J.; Whitesides, G. M. Oriented Growth of Calcite Controlled by Self-Assembled Monolayers of Functionalized Alkanethiols Supported on Gold and Silver. J. Am. Chem. Soc. 1999, 121, 4500-4509.

(42) Travaille, A. M.; Donners, J. J. J. M.; Gerritsen, J. W.; Sommerdijk, N. A. J. M.; Nolte, R. J. M.; Van Kempen, H. Aligned Growth of Calcite Crystals on a Self-Assembled Monolayer. Adv. Mater. 2002, 14, 492-495.

(43) Ghosh, M.; Yang, D. S. Structures of Self-Assembled n-Alkanethiols on Gold by Reflection High-Energy Electron Diffraction. Phys. Chem. Chem. Phys. 2020, 22, 1732517335 .

(44) Camillone, N.; Chidsey, C. E. D.; Liu, G. Y.; Putvinski, T. M.; Scoles, G. Surface Structure and Thermal Motion of N-Alkane Thiols Self-Assembled on Au(111) Studied by Low Energy Helium Diffraction. J. Chem. Phys. 1991, 94, 8493-8502.

(45) Schönenberger, C.; Sondag-Huethorst, J. A. M.; Jorritsma, J.; Fokkink, L. G. J. What Are the "Holes" in Self-Assembled Monolayers of Alkanethiols on Gold? Langmuir 1994, 10, 611-614.

(46) Poirier, G. E. Mechanism of Formation of Au Vacancy Islands in Alkanethiol Monolayers on Au(111). Langmuir 1997, 13, 2019-2026.

(47) Warren, B. E. (Bertram E. X-Ray Diffraction; Dover Publications, 1990.

(48) Kirchner, M. T.; Das, D.; Boese, R. Cocrystallization with Acetylene: Molecular Complex with Methanol. Cryst. Growth Des. 2008, 8, 763-765. 
(49) Smith, R. S.; Tylinski, M.; Kimmel, G. A.; Kay, B. D. Crystallization Kinetics of Amorphous Acetonitrile Nanoscale Films. J. Chem. Phys. 2021, 154, 144703.

(50) Lin, T. J.; Hsing, C. R.; Wei, C. M.; Kuo, J. L. Structure Prediction of the Solid Forms of Methanol: An Ab Initio Random Structure Searching Approach. Phys. Chem. Chem. Phys. 2016, 18, 2736-2746. 


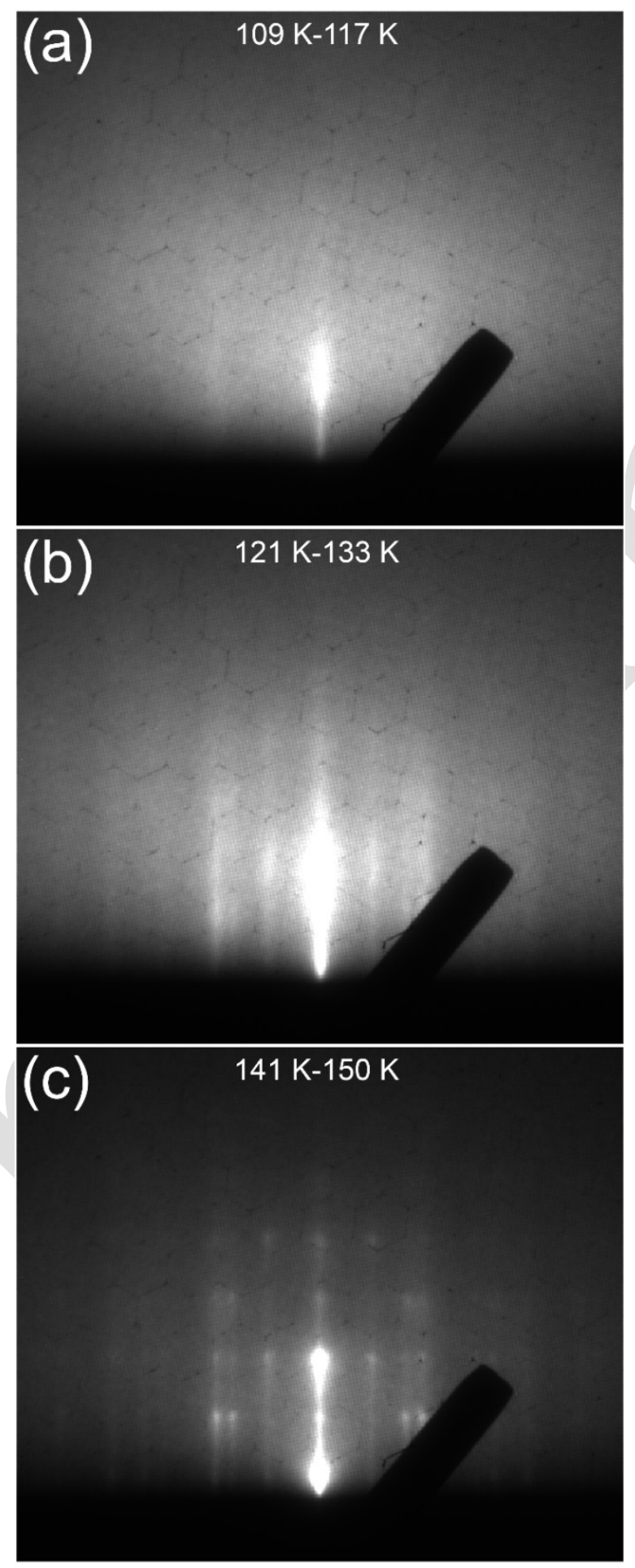

Figure S1. RHEED images of 5-nm ethanol films obtained in the annealing temperature range of (a) $109-117 \mathrm{~K}$, (b) $121-133 \mathrm{~K}$, and (c) $141-150 \mathrm{~K}$. 

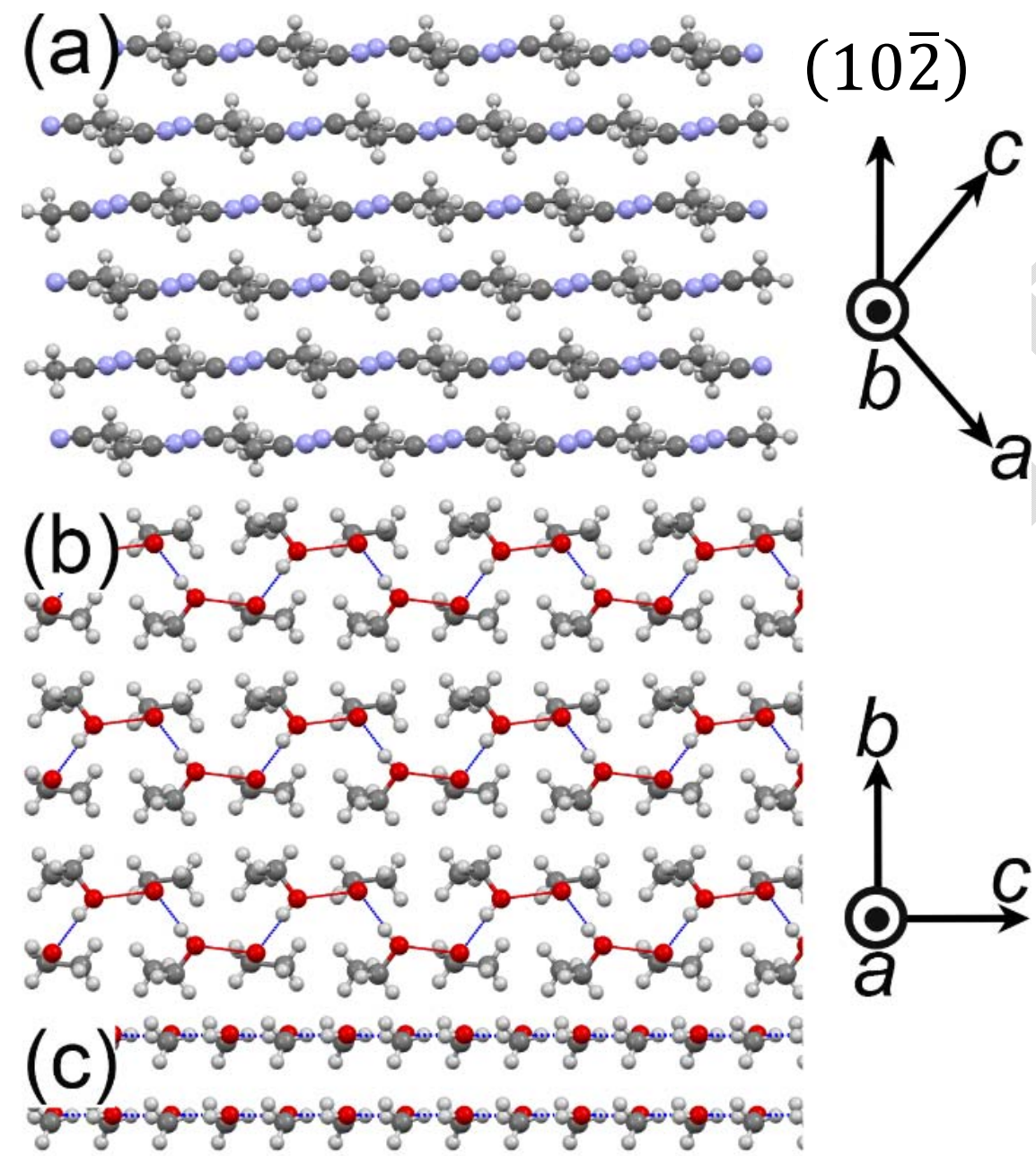

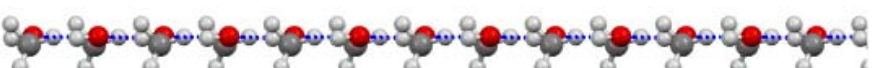

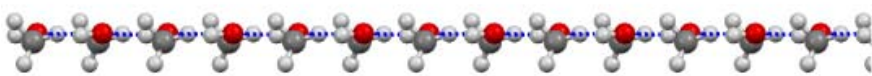
80.

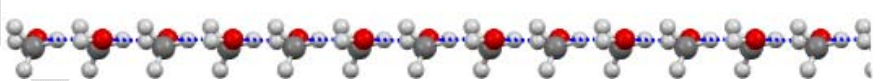
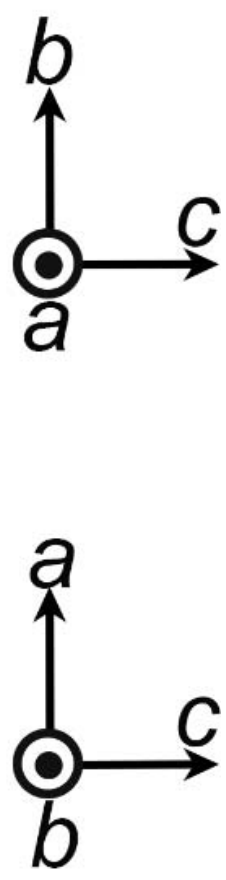

Figure S2. Side view of the molecular crystal structures formed on the hydrophobic SAM surface: (a) Monoclinic a phase of acetonitrile, (b) monoclinic $\alpha$ phase of ethanol, and (c) orthorhombic $\beta$ phase of methanol. The carbon, nitrogen, oxygen, and hydrogen atoms are in gray, light blue, red, and white, respectively. The blue dashed lines in (b) and (c) indicate the hydrogen bonds. 

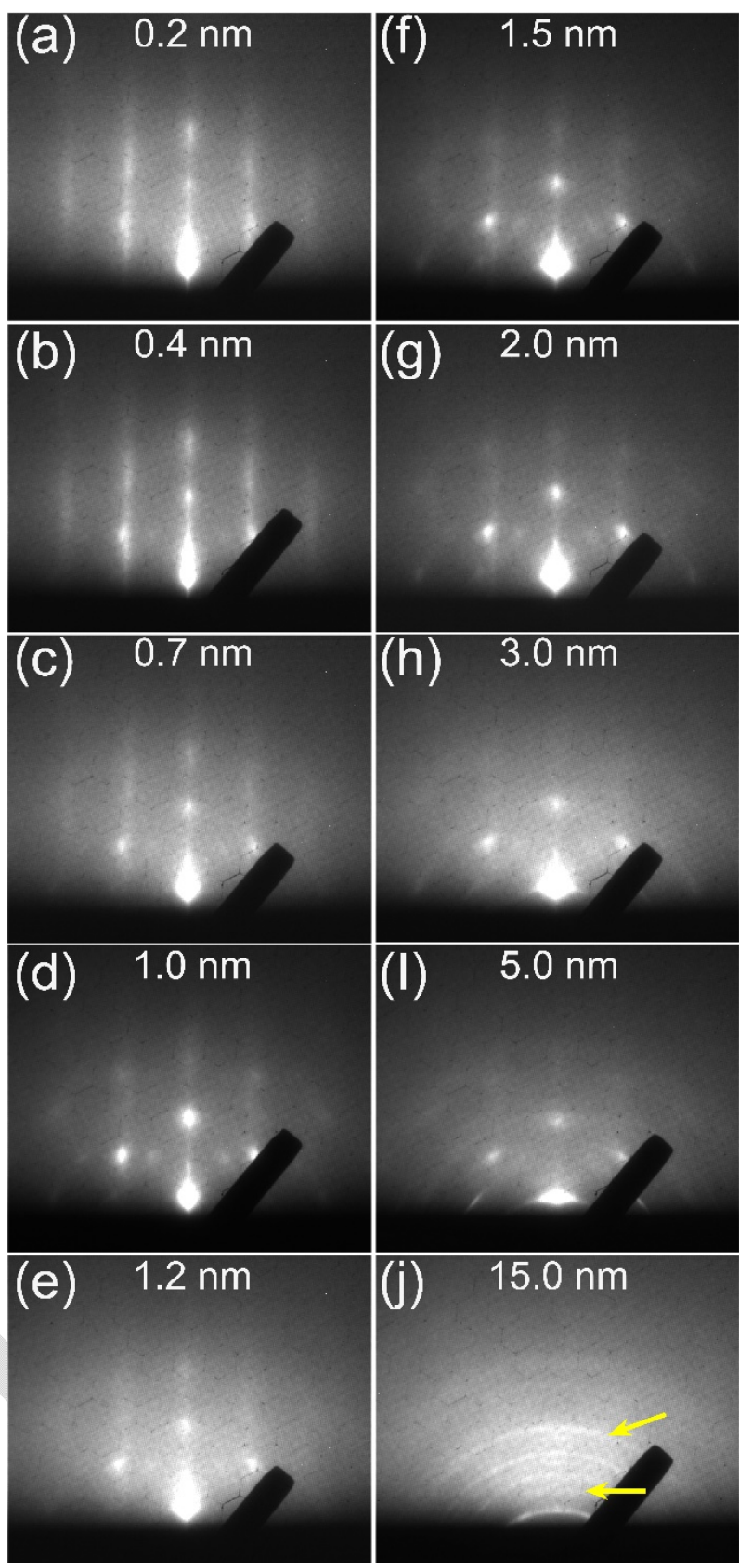

Figure S3. RHEED images of methanol thin films on the hydrophobic SAM surface with different nominal thicknesses. (a-e) Increasing intensities of the Bragg spots from crystalline methanol with decreasing intensities of the SAM diffraction features. The methanol Bragg spots signify the formation of $3 \mathrm{D}$ crystalline islands. (f-j) Conversion of the Bragg spots into diffraction rings in a relatively thick film $(15 \mathrm{~nm})$, signifying the loss of the epitaxial ordering and the original phase. The two arrows in (j) indicate the diffractions that are present in the orthorhombic $\alpha$ phase but absent in orthorhombic $\beta$ phase. 


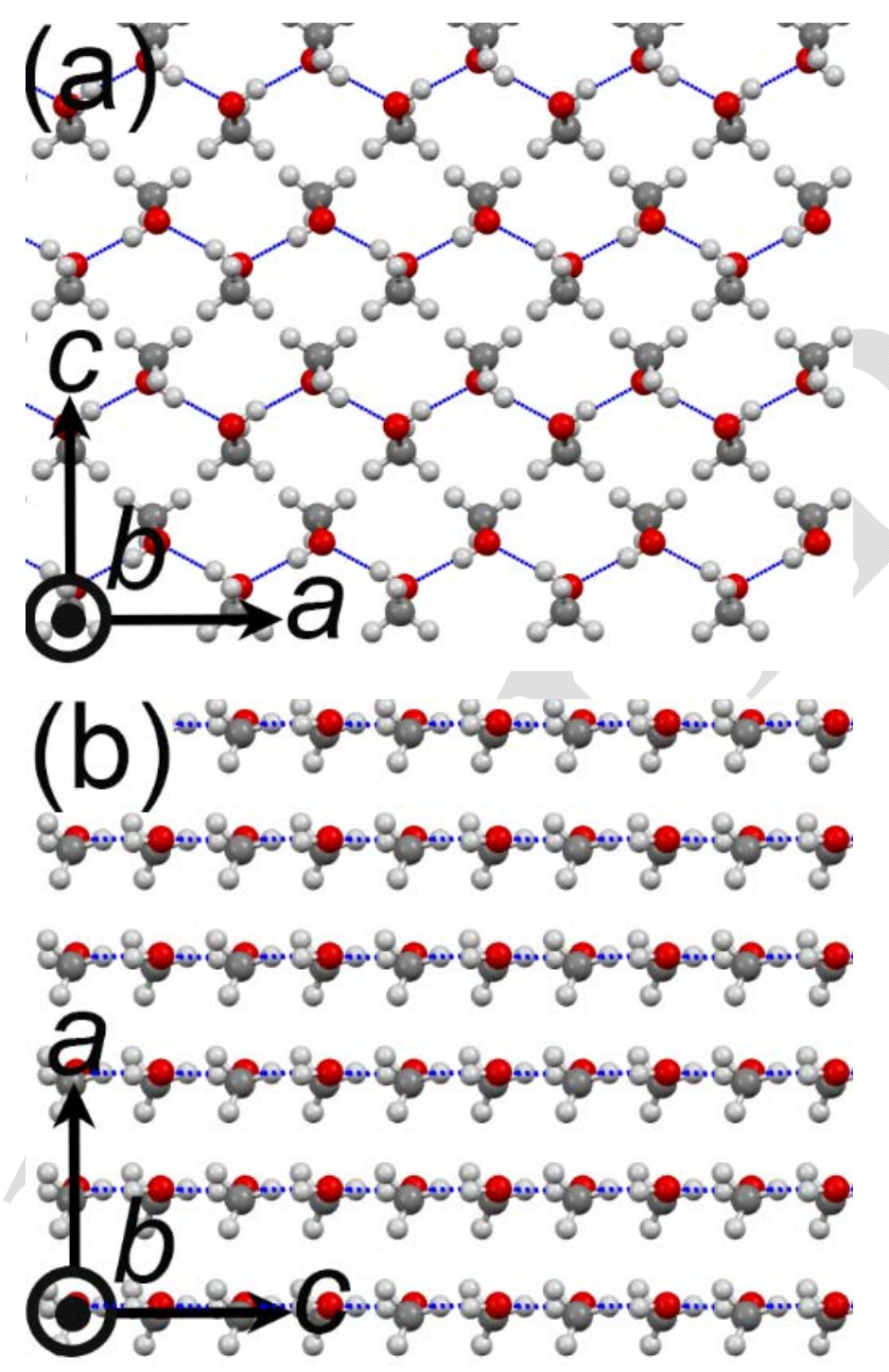

Figure S4. Side view of the two different phases of solid methanol: (a) orthorhombic $\alpha$ phase and (b) orthorhombic $\beta$ phase. The carbon, oxygen, and hydrogen atoms are in gray, red, and white, respectively. The blue dashed lines indicate the hydrogen bonds. 